BELLE Preprint 2002-3

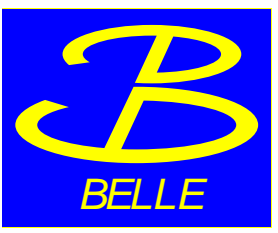

KEK Preprint 2001-168

\title{
Results of time evolution analyses of $B$-decays at Belle
}

\author{
Y. Sakai \\ High Energy Accelerator Research Organization (KEK) \\ 1-1, Oho, Tsukuba-shi, Ibaraki-ken, Japan 305-0801
}

\begin{abstract}
We report results of proper-time evolution analyses of $B$ decays at Belle, based on a $29.1 \mathrm{fb}^{-1}$ data sample recorded at the $\Upsilon(4 S)$ resonance. These results include measurements of $\sin 2 \phi_{1}$, the lifetime of $B$-mesons, and the mass-difference between two mass eigenstates of the $B^{0}-\bar{B}^{0}$ system $\left(\Delta m_{d}\right) . \Delta m_{d}$ is measured using three different methods: fully reconstructed hadronic modes, a fully reconstructed semi-leptonic mode, and a partially reconstructed $D^{* \pm} \pi^{\mp}$ mode.
\end{abstract}

Contributed to the Proceedings of the

Fifth KEK Topical Conference, 20-22, Nov. 2001, Tsukuba, Japan 


\section{Introduction}

The main goal of a $B$-factory is to first observe and establish $C P$ violation in $B$-decays and then to precisely measure the $C P$ violation angles of the Unitarity Triangle, as well as the lengths of its sides. The aims is to either confirm the Kobayashi-Maskawa model[1] for the mechanism of $C P$ violation or find evidence of physics beyond the Standard Model. Analysis of the proper-time evolution of $B$ decays is essential for measuring mixinginduced $C P$ violation, and is one of the unique features of an asymmetric $e^{+} e^{-}$collider at the $\Upsilon(4 S)$. By combining a large number of accumulated $B$ events with a clean environment to study $B$-decays, the asymmetric-collider experiments can now provide the most precise measurements that involve proper-decay time analyses, such as lifetimes and the oscillation frequency in $B^{0}-\bar{B}^{0}$ mixing, which is characterized by the mass-difference between the two mass eigenstates of the $B^{0}-\bar{B}^{0}$ system $\left(\Delta m_{d}\right)$.

In this article, we report on the results of proper-time analyses of $B$ decays at Belle: measurements of $\sin 2 \phi_{1}[2]$, the lifetime of $B$-mesons, and $\Delta m_{d}$.

\section{KEKB Accelerator and Belle}

KEKB [3] is an asymmetric $e^{+} e^{-}$collider of $3 \mathrm{~km}$ circumference, which consists of $8 \mathrm{GeV} e^{-}$and $3.5 \mathrm{GeV} e^{+}$storage rings and an injection linear accelerator for them. It has one interaction point where the $e^{+}$and $e^{-}$beams collide with a finite crossing angle of $22 \mathrm{mrad}$. The collider has been operated with peak beam currents of $1230 \mathrm{~mA}\left(e^{+}\right)$and $780 \mathrm{~mA}\left(e^{-}\right)$, giving a peak luminosity of $5.5 \times 10^{33} / \mathrm{cm}^{2} / \mathrm{sec}$ (as of the end of 2001 , the target is $\left.10^{34} / \mathrm{cm}^{2} / \mathrm{sec}\right)$. Due to the energy asymmetry, the $\Upsilon(4 S)$ and its daughter $B$-pair are produced with $\beta \gamma=0.425$ along the electron beam direction ( $\mathrm{z}$ direction) in the laboratory frame. The average distance between the two decay vertices of $B$ mesons is approximately $200 \mu \mathrm{m}$. A total integrated luminosity of $47 \mathrm{fb}^{-1}$ was accumulated during the period between October 1999 and the end of 2001, of which $4.0 \mathrm{fb}^{-1}$ was taken off-resonance and the rest was taken on the $\Upsilon(4 S)$. In the analyses reported here, we use a 29.1 $\mathrm{fb}^{-1}$ data sample, which contains 31.3 million $B \bar{B}$ pairs, recorded at $\Upsilon(4 S)$ between January 2000 and July 2001.

Belle is an international collaboration consisting of $\sim 300$ physicists from $\sim 50$ institutes in 14 countries (U.S.A., Russia, Asia, Australia, and Europe). 
The Belle detector 41 is a general purpose large solid angle magnetic spectrometer surrounding the interaction point.

Charged particle tracking is done by a silicon vertex detector (SVD) and a central drift chamber (CDC). The SVD consists of three layers of doublesided silicon strip detectors (DSSD) at radii of 3.0, 4.5 and $6.0 \mathrm{~cm}$. The CDC is a small-cell cylindrical drift chamber consisting of 50 layers of anode wires (18 stereo wire layers), covering $17^{\circ}<\theta_{l a b}<150^{\circ}$. The CDC is operated with a $\mathrm{He}(50 \%)+\mathrm{C}_{2} \mathrm{H}_{6}(50 \%)$ mixture. The CDC also provides measurements of the energy loss with a resolution of $\sigma(d E / d x)=6.9 \%$.

Particle identification is done by three detectors: $d E / d x$ measurements in the CDC, time-of-flight counters (TOF) and aerogel Cherenkov counters (ACC). The TOF system consists of 128 plastic scintillators. The time resolution is 95 psec $(\mathrm{rms})$. The ACC consists of 1188 aerogel blocks with refractive indices of between 1.01 and 1.03, depending on the polar angle. By combining information from these detectors, the efficiency for $K^{ \pm}$identification is about $90 \%$ and the $\pi$ fake rate is $6 \%$ with the requirement $P(K / \pi)>0.6$.

The electromagnetic calorimeter (ECL) consists of $8736 \mathrm{CsI}(\mathrm{Tl})$ crystal blocks, 16.1 radiation length thick, and covering the same angular region as CDC. Electron identification in Belle is based on a combination of $d E / d x$ measurements in the CDC, the response of the ACC, and the position, shape and total energy (i.e. $E / p$ ) of its associated CsI shower.

The outermost detector, for the measurement of $\mu^{ \pm}$and $K_{L}$ (KLM), consists of 14 layers of iron ( $4.7 \mathrm{~cm}$ thick) absorbers alternating with resistive plate counters (RPC).

\section{Measurement of $\sin 2 \phi_{1}$}

The Standard Model (SM) predicts a $C P$ violating asymmetry in the timedependent rates for the initial $B^{0}$ and $\bar{B}^{0}$ decays to a common $C P$ eigenstate, $f_{C P}$. In the case where $f_{C P}=(c \bar{c}) K^{0}$, the asymmetry is given by

$$
\begin{aligned}
A(\Delta t) & \equiv \frac{R\left(\bar{B}^{0} \rightarrow f_{C P}\right)-R\left(B^{0} \rightarrow f_{C P}\right)}{R\left(\bar{B}^{0} \rightarrow f_{C P}\right)+R\left(B^{0} \rightarrow f_{C P}\right)} \\
& =-\xi_{f} \sin 2 \phi_{1} \sin \left(\Delta m_{d} \Delta t\right),
\end{aligned}
$$

where $\xi_{f}$ is the $C P$-eigenvalue of $f_{C P}, \Delta t$ is proper-time difference of two $B$ decays, and $\phi_{1}$ is one of the three internal angles of the Unitarity Triangle, 
defined as $\phi_{1} \equiv \pi-\arg \left(\frac{-V_{t b}^{*} V_{t d}}{-V_{c b}^{*} V_{c d}}\right)(\equiv \beta)[$ [ $]$.

The measurement requires the reconstruction of $B^{0} \rightarrow f_{C P}$ decays, the determination of the $b$-flavor of the accompanying $B$-decay $\left(f_{\text {tag }}\right)$, a measurement of $\Delta t$, and a fit of the expected $\Delta t$ distribution to the measured distribution using a maximum-likelihood method.

We reconstruct $B^{0}$ decays to the following $C P$ eigenstates [6]: $J / \psi K_{S}$, $\psi(2 S) K_{S}, \chi_{c 1} K_{S}, \eta_{c} K_{S}$ for $\xi_{f}=-1$ and $J / \psi K_{L}$ for $\xi_{f}=+1$. The $J / \psi$ and $\psi(2 S)$ mesons are reconstructed via their decays to $\ell^{+} \ell^{-}(\ell=\mu, e)$. The $\psi(2 S)$ is also reconstructed via its $J / \psi \pi^{+} \pi^{-}$decay, the $\chi_{c 1}$ via its $J / \psi \gamma$ decay, and the $\eta_{c}$ via its $K^{+} K^{-} \pi^{0}$ and $K_{S}\left(\pi^{+} \pi^{-}\right) K^{-} \pi^{+}$decays. We also use $B^{0} \rightarrow J / \psi K^{* 0}\left(K_{S} \pi^{0}\right)$ decays, where the final state is a mixture of even and odd $C P$. We find that the final state is primarily $\xi_{f}=+1$; the $\xi_{f}=-1$ fraction is $0.19 \pm 0.04$ (stat) \pm 0.04 (syst) [7].

Except for $B^{0} \rightarrow J / \psi K_{L}$, we identify $B$ decays using the energy difference $\Delta E \equiv E_{B}^{\mathrm{cms}}-E_{\mathrm{beam}}^{\mathrm{cms}}$ and the beam-energy constrained mass $M_{\mathrm{bc}} \equiv$ $\sqrt{\left(E_{\text {beam }}^{\mathrm{cms}}\right)^{2}-\left(p_{B}^{\mathrm{cms}}\right)^{2}}$, where $E_{\text {beam }}^{\mathrm{cms}}$ is the cms beam energy, and $E_{B}^{\mathrm{cms}}$ and $p_{B}^{\mathrm{cms}}$ are the cms energy and momentum of the $B$ candidate. The $B$ meson signal region is defined as $5.270<M_{\mathrm{bc}}<5.290 \mathrm{GeV} / c^{2}$ and a mode-dependent window around $\Delta E=0$. Table 1 lists the numbers of observed candidates $\left(N_{\text {ev }}\right)$ and the background $\left(N_{\text {bkgd }}\right)$ determined by extrapolating the rate in the non-signal $\Delta E$ vs. $M_{\mathrm{bc}}$ region into the signal region.

Table 1: Numbers of observed events $\left(N_{\mathrm{ev}}\right)$ and the estimated background $\left(N_{\text {bkgd }}\right)$ in the signal region for each $f_{C P}$ mode.

\begin{tabular}{lrr}
\hline Mode & $N_{\text {ev }}$ & $N_{\text {bkgd }}$ \\
\hline$J / \psi\left(\ell^{+} \ell^{-}\right) K_{S}\left(\pi^{+} \pi^{-}\right)$ & 457 & 11.9 \\
$J / \psi\left(\ell^{+} \ell^{-}\right) K_{S}\left(\pi^{0} \pi^{0}\right)$ & 76 & 9.4 \\
$\psi(2 S)\left(\ell^{+} \ell^{-}\right) K_{S}\left(\pi^{+} \pi^{-}\right)$ & 39 & 1.2 \\
$\psi(2 S)\left(J / \psi \pi^{+} \pi^{-}\right) K_{S}\left(\pi^{+} \pi^{-}\right)$ & 46 & 2.1 \\
$\chi_{c 1}(J / \psi \gamma) K_{S}\left(\pi^{+} \pi^{-}\right)$ & 24 & 2.4 \\
$\eta_{c}\left(K^{+} K^{-} \pi^{0}\right) K_{S}\left(\pi^{+} \pi^{-}\right)$ & 23 & 11.3 \\
$\eta_{c}\left(K_{S} K^{-} \pi^{+}\right) K_{S}\left(\pi^{+} \pi^{-}\right)$ & 41 & 13.6 \\
$J / \psi K^{* 0}\left(K_{S} \pi^{0}\right)$ & 41 & 6.7 \\
\hline Sub-total & 747 & 58.6 \\
\hline$J / \psi\left(\ell^{+} \ell^{-}\right) K_{L}$ & 569 & 223 \\
\hline
\end{tabular}

Candidate $B^{0} \rightarrow J / \psi K_{L}$ decays are selected by requiring ECL and/or 
KLM hit patterns that are consistent with the presence of a shower induced by a neutral hadron. The quantity $p_{B}^{\mathrm{cms}}$ is calculated with the $B^{0} \rightarrow J / \psi K_{L}$ two-body decay hypothesis. The $p_{B}^{\mathrm{cms}}$ distribution is fitted to the sum of signal and background distributions. There are 569 entries in the $0.2 \leq$ $p_{B}^{\mathrm{cms}} \leq 0.45(0.40) \mathrm{GeV} / c$ signal region with KLM (ECL) clusters.

Tracks that are not associated with a reconstructed $C P$ eigenstate decay are used for flavor tagging. Initially, the $b$-flavor determination is performed at the track level: high momentum leptons from $b \rightarrow c \ell^{-} \bar{\nu}$, lower momentum leptons from $c \rightarrow s \ell^{+} \nu$, charged kaons and $\Lambda$ baryons from $b \rightarrow c \rightarrow s$, high momentum pions that originate from decays of $B^{0} \rightarrow D^{(*)-}(n \pi)^{+}$, and slow pions from $D^{*+} \rightarrow D^{0} \pi^{+}$. We use the MC to determine a category-dependent variable that ranges from -1 for a reliably identified $\bar{B}^{0}$ to +1 for a reliably identified $B^{0}$, and depends on the tagging particle's charge, cms momentum, polar angle and particle-identification probability, as well as other kinematic and event shape quantities. The results from the separate track categories are then combined to take into account correlations in the case of multiple track-level tags. This stage determines two event-level parameters, $q$ and $r$ : $q$ takes values $q=+1(-1)$ when the tag-side $B$ meson is more likely to be a $B^{0}\left(\bar{B}^{0}\right) ; r$ is an event-by-event flavor-tagging dilution factor, which ranges from $r=0$ for no flavor discrimination to $r=1$ for unambiguous flavor assignment. It is used only to sort data into six intervals of $r$, according to the flavor purity.

The wrong-tag probabilities, $w_{l}(l=1,6)$, are determined directly from the data for the six $r$ intervals using fully reconstructed, self-tagged decays, as described in Sec. 5.1. The total effective tagging efficiency is $\sum_{l} f_{l}\left(1-2 w_{l}\right)^{2}=$ $0.270 \pm 0.008$ (stat) ${ }_{-0.009}^{+0.006}$ (syst), where $f_{l}$ is the fraction of the events in each $r$ interval.

The vertex positions for the $f_{C P}$ and $f_{\text {tag }}$ decays are reconstructed using tracks that have at least one three-dimensional coordinate determined from associated $r-\phi$ and $z$ hits in the same SVD layer along with one or more additional $z$ hits in the other layers. Each vertex position is required to be consistent with the interaction point profile smeared in the $r-\phi$ plane by the $B$ meson decay length. The $f_{C P}$ vertex is determined using lepton tracks from $J / \psi$ or $\psi(2 S)$ decays, or prompt tracks from $\eta_{c}$ decays. The $f_{\text {tag }}$ vertex is determined from well reconstructed tracks not assigned to $f_{C P}$. Tracks that form a $K_{S}$ are not used.

We determine $\sin 2 \phi_{1}$ by performing an unbinned maximum-likelihood fit of a probability density function (pdf) to the observed $\Delta t$ distributions. 
The pdf is a convolution of the theoretical distribution with a $\Delta t$ resolution function, including the effect the of background and wrong-tag probabilities. The result is

$$
\sin 2 \phi_{1}=0.99 \pm 0.14 \text { (stat) } \pm 0.06 \text { (syst). }
$$

The systematic error is dominated by the uncertainties in vertex reconstruction and resolution. Figure 1 shows the observed $\Delta t$ distributions for the $q \xi_{f}=+1$ (solid points) and $q \xi_{f}=-1$ (open points) event samples. In

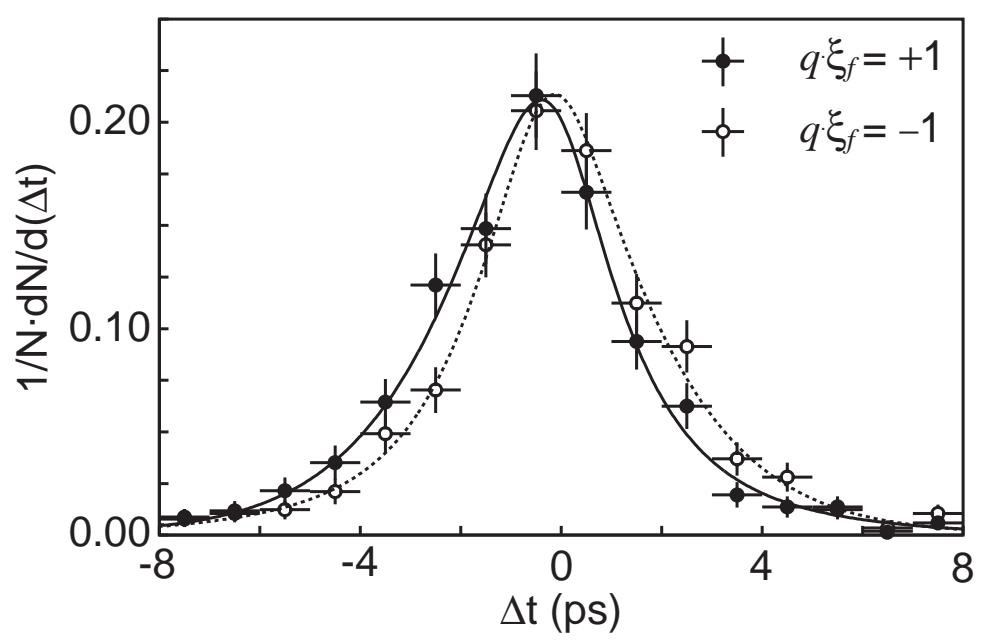

Figure 1: $\Delta t$ distributions for the events with $q \xi_{f}=+1$ (solid points) and $q \xi_{f}=-1$ (open points). The results of the global fit (with $\sin 2 \phi_{1}=0.99$ ) are shown as solid and dashed curves, respectively.

Fig. 2(a) we show the asymmetries for the combined data sample that are obtained by applying the fit to the events in each $\Delta t$ bin separately. The smooth curve is the result of the global unbinned fit. Figures 2(b) and (c) show the corresponding asymmetries for the $(c \bar{c}) K_{S}\left(\xi_{f}=-1\right)$ and the $J / \psi K_{L}$ $\left(\xi_{f}=+1\right)$ modes separately. The fits give $\sin 2 \phi_{1}$ values to be $0.84 \pm 0.17$ (stat) and $1.31 \pm 0.23$ (stat), respectively, which are opposite, as expected. Fitting to the non- $C P$ eigenstate self-tagged modes $B^{0} \rightarrow D^{(*)-} \pi^{+}, D^{*-} \rho^{+}$, $J / \psi K^{* 0}\left(K^{+} \pi^{-}\right)$and $D^{*-} \ell^{+} \nu$, yields $0.05 \pm 0.04$. As shown in Fig. 2(d), no asymmetry is seen, as expected. 


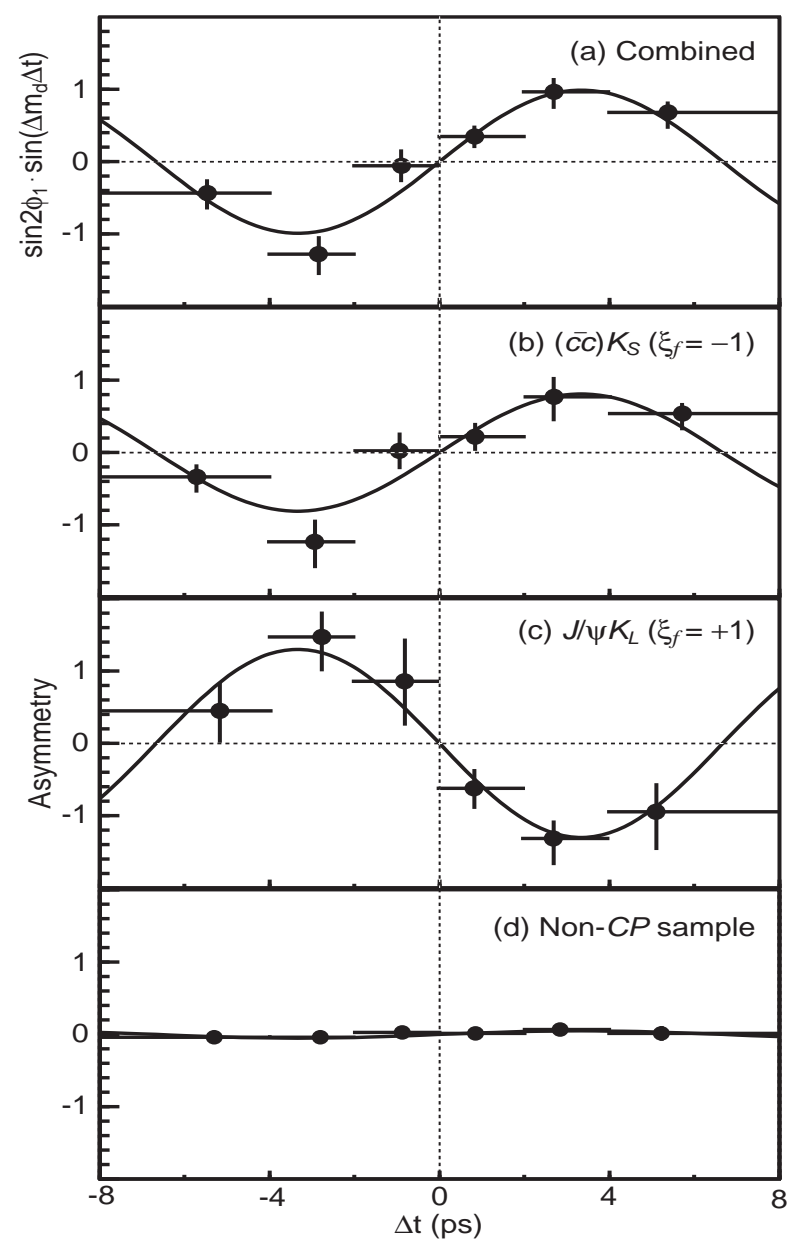

Figure 2: (a) The asymmetry obtained from separate fits to each $\Delta t$ bin for the full data sample; the curve is the result of the global fit. The corresponding plots for the (b) $(c \bar{c}) K_{S}\left(\xi_{f}=-1\right)$, (c) $J / \psi K_{L}\left(\xi_{f}=+1\right)$, and (d) $B^{0}$ control samples are also shown. The curves are the results of the fit applied separately to the individual data samples. 


\section{$4 \quad$ Lifetime Measurements}

For lifetime measurements, $\bar{B}^{0}$ and $B^{-}$mesons are fully reconstructed in the following hadronic decay modes: $\bar{B}^{0} \rightarrow D^{+} \pi^{-}, D^{*+} \pi^{-}, D^{*+} \rho^{-}, J / \psi K_{S}$, $J / \psi K^{* 0}\left(K^{-} \pi^{+}\right)$, and $B^{-} \rightarrow D^{0} \pi^{-}, J / \psi K^{-}$. The reconstruction of $J / \psi X$ is made in the way quite similar to that used for the $C P$ analysis described above. Neutral and charged $D$ candidates are reconstructed in the following channels: $D^{0} \rightarrow K^{-} \pi^{+}, K^{-} \pi^{+} \pi^{0}, K^{-} \pi^{+} \pi^{+} \pi^{-}$, and $D^{+} \rightarrow K^{-} \pi^{+} \pi^{+}$. $D^{*+} \rightarrow D^{0} \pi^{+}$candidates are formed by combining a $D^{0}$ candidate with a positively charged soft pion. To reduce the continuum background, a selection based on the ratio of the second to zeroth Fox-Wolfram moments 8 and the angle between the thrust axes of the reconstructed and associated $B$ mesons is applied mode by mode. The vertices for fully reconstructed and associated $B$ 's are reconstructed using the same algorithm that is used in the $C P$ analysis. We find $7863 \bar{B}^{0}$ and $12047 B^{-}$events within the $\Delta E-M_{\mathrm{bc}}$ signal regions after the vertexing and selections are applied.

We extract the $\bar{B}^{0}$ and $B^{-}$lifetimes by performing an unbinned maximumlikelihood fit to the $\Delta t$ distributions of $\bar{B}^{0}$ and $B^{-}$simultaneously. The pdf is similar to the one used in the $C P$ analysis. However, we have developed a more elaborate resolution function for lifetime measurements. It is constructed as the convolution of four different contributions: the detector resolutions for the fully reconstructed $B$ and associated $B$, the additional smearing from the associated $B$ due to the inclusion of secondary tracks and the kinematic approximation that the $B$ mesons are at rest in the cms. We allow for outliers with a large-width Gaussian to take into account any imperfection of the resolution function. The fit includes 10 free parameters $(7$ for resolution function and 3 for outliers) besides $\tau_{\bar{B}^{0}}$ and $\tau_{B^{-}}$. We obtain 9 :

$\tau_{\bar{B}^{0}}=1.554 \pm 0.030$ (stat) \pm 0.019 (syst) ps,

$\tau_{B^{-}}=1.695 \pm 0.026$ (stat) \pm 0.015 (syst) ps,

$\tau_{B^{-}} / \tau_{\bar{B}^{0}}=1.091 \pm 0.023$ (stat) \pm 0.014 (syst).

Figure 3 shows the distributions of $\Delta t$ for $\bar{B}^{0}$ and $B^{-}$events in the signal region with the fitted curves superimposed. The dominant sources of the systematic errors are the uncertainties in the background $\Delta t$ shape and the modeling of resolution function. 


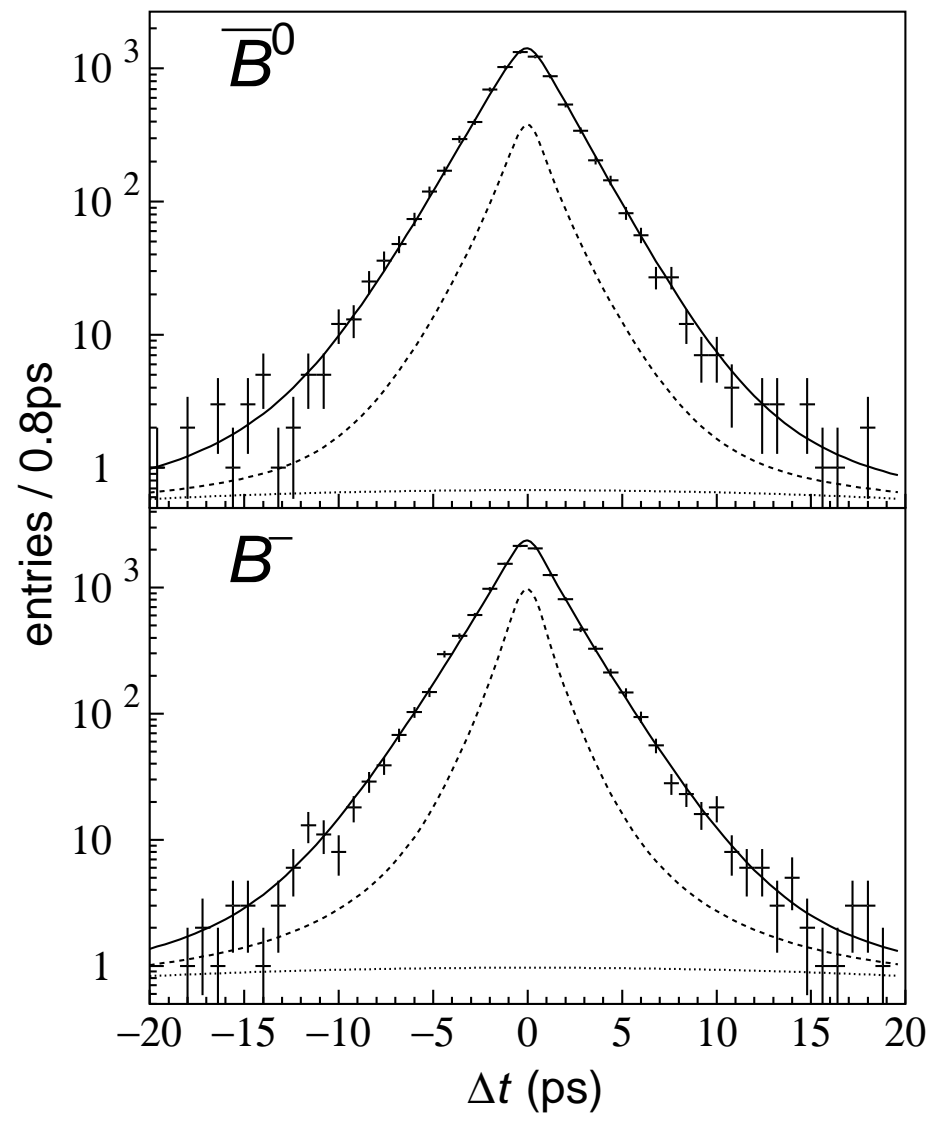

Figure 3: $\Delta t$ distributions of neutral (top) and charged (bottom) $B$ meson pairs with fitted curves. The dashed lines represent the sum of the background and the outlier component, and the dotted lines represent the outlier component.

\section{$5 \Delta m_{d}$ Measurements}

$\Delta m_{d}$ can be measured from the oscillation pattern of the $\Delta t$ distributions of the mixed (same flavor, SF) and unmixed (opposite flavor, OF) $B$ pairs:

$$
\begin{aligned}
& N_{\mathrm{SF}} \propto e^{-|\Delta t| / \tau_{B^{0}}}\left[1-\cos \left(\triangle m_{d} \Delta t\right)\right], \\
& N_{\mathrm{OF}} \propto e^{-|\Delta t| / \tau_{B^{0}}}\left[1+\cos \left(\triangle m_{d} \Delta t\right)\right] .
\end{aligned}
$$

We published a result using inclusive dileptons based on an early data 
sample 10]. Here, we report on the results using three other different samples: fully reconstructed hadronic modes, a fully reconstructed semi-leptonic mode, and a partially reconstructed $D^{* \pm} \pi^{\mp}$ mode.

\subsection{Fully Reconstructed Modes}

We use fully reconstructed, self-tagged decay modes in two categories: hadronic decays $\left(D^{(*)-} \pi^{+}, D^{*-} \rho^{+}\right)$and semi-leptonic decay $\left(B^{0} \rightarrow D^{*-} \ell^{+} \nu\right)$. For hadronic modes, the same event samples are used as lifetime measurements. For $B^{0} \rightarrow D^{*-} \ell^{+} \nu$, we use the same $D^{*-}$ decay chains as for the hadronic modes. The $D^{*-}$ candidates are combined with $\mu^{+}$or $e^{+}$candidates having charge opposite to that of the $D^{*-}$ candidate. We exploit the massless character of the neutrino using the missing-mass squared in the cms defined by $M_{\text {miss }}^{2}=M M-C \cos \theta_{B, D^{*} \ell}$ where $M M=\left(E_{B}-E_{D^{*} \ell}\right)^{2}-\left|\vec{p}_{B}\right|^{2}-\left|\vec{p}_{D^{*} \ell}\right|^{2}$, $C=2\left|\vec{p}_{B}\right|\left|\vec{p}_{D^{*} \ell}\right| . \quad E_{B}$ and $\vec{p}_{B}\left(E_{D^{*} \ell}\right.$ and $\left.\vec{p}_{D^{*} \ell}\right)$ are cms energy and momentum of $B^{0}$ ( $D^{*}$ and lepton system). $\theta_{B, D^{*} \ell}$ is an angle between vectors $\vec{p}_{B}$ and $\vec{p}_{D^{*} \ell}$. It gives $\cos \theta_{B, D^{*} \ell}=-M M / C$ by setting $M_{\text {miss }}^{2}=0$. We select $D^{*-} \ell^{+} \nu$ candidates by requiring $\left|\cos \theta_{B, D^{*} \ell}\right|<1.1$. The $b$-flavor of the accompanying $B$ meson is assigned according to the flavor-tagging algorithm described above. The values of $w_{l}$ are obtained from the amplitudes of the time-dependent $B^{0} \bar{B}^{0}$ mixing oscillations: $\left(N_{\mathrm{OF}}-N_{\mathrm{SF}}\right) /\left(N_{\mathrm{OF}}+N_{\mathrm{SF}}\right)=$ $\left(1-2 w_{l}\right) \cos \left(\Delta m_{d} \Delta t\right)$. We perform a maximum-likelihood fit to $\mathrm{SF}$ and $\mathrm{OF}$ $\Delta t$ distributions simultaneously with $\Delta m_{d}$ and $w_{l}$ 's as free parameters. $\left(\Delta m_{d}\right.$ is fixed at the world average value [11] when we determine $w_{l}$ 's.) We obtain $\Delta m_{d}=0.521 \pm 0.017$ (stat) ${ }_{-0.014}^{+0.011}$ (syst) $\mathrm{ps}^{-1}$ (prelim.) for hadronic modes and $\Delta m_{d}=0.489 \pm 0.012$ (stat) ${ }_{-0.014}^{+0.011}$ (syst) $\mathrm{ps}^{-1}$ (prelim.) for semi-leptonic mode. Dominant sources of systematic errors for hadronic modes are uncertainties in the resolution function and the background components, including mixing. Those for the semi-leptonic mode are uncertainties in the effects of the backgrounds.

\subsection{Partially Reconstructed $D^{* \pm} \pi^{\mp}$}

In this method, $B^{0} \rightarrow D^{* \pm} \pi^{\mp}$ decays are reconstructed using only the fast pion $\left(\pi_{f}\right)$ directly from the $B^{0}$ and the soft pion $\left(\pi_{s}\right)$ from $D^{*+} \rightarrow D^{0} \pi^{+}$. Here, we use a method based on reconstructing the $D^{0}$ missing mass. We select a $\pi_{f}$ with cms momentum $2.05<p_{\pi_{f}}^{*}<2.45 \mathrm{GeV} / \mathrm{c}$ and an oppositely charged $\pi_{s}$ with a cms momentum of less than $0.45 \mathrm{GeV} / \mathrm{c}$. The missing 
mass is then calculated assuming the $B$ meson is at rest in the cms. We select events with missing mass larger than $1.85 \mathrm{GeV} / \mathrm{c}^{2}$ as $D^{* \pm} \pi^{\mp}$ candidates. We require $R_{2}$ to be less than 0.5 to reduce the continuum background. In order to identify the flavor of the other $B$, we require an additional high momentum lepton with a cms momentum larger than $1.1 \mathrm{GeV} / \mathrm{c}$. To reduce the background from incorrect tags (i.e. $\pi_{f}$ and leptons from the same $B$ ), we further require $\cos \theta_{\ell \pi_{f}}^{*}>-0.8$ where $\theta_{\ell \pi_{f}}^{*}$ is the angle between a $\pi_{f}$ and a lepton in the cms. We select $3686 \mathrm{OF}$ (1213 SF) candidates with $73 \%(62 \%)$ purity after the missing mass cut and lepton-tag. The $z$ vertex of leptons is determined from the intersection of the lepton tracks with the profile of $B_{d}^{0}$ decay vertices. An unbinned maximum likelihood fit is performed to the $\mathrm{OF}$ and $\mathrm{SF} \Delta z$ distributions to extract $\Delta m_{d}$. We obtain $\Delta m_{d}=0.505 \pm 0.017$ (stat) \pm 0.020 (syst) $\mathrm{ps}^{-1}$ (prelim.). The dominant sources of systematic errors are uncertainties in the background fraction and the resolution function.

Figure 1 shows the OF-SF asymmetries as a function of $\Delta z$ for each method mentioned above together with the fit curves.

\section{Conclusion}

Belle (along with BaBar) presented the first significant observation of the $C P$ violation in 2001 summer. The measured value is

$\sin 2 \phi_{1}=0.99 \pm 0.14$ (stst) \pm 0.06 (syst)

with more than $6 \sigma$ significance. We have also measured the lifetimes of $\bar{B}^{0}$ and $B^{-}$and $\Delta m_{d}$. The results are consistent with those of other experiments [12]13. We find $\tau_{B^{-}} / \tau_{\bar{B}^{0}}$ is different from 1 with more than $3 \sigma$ significance. By combining four different methods, we obtain $\Delta m_{d}=$

$0.490 \pm 0.010 \mathrm{ps}^{-1}$ (prelim.). These are the most precise measurements so far.

\section{Acknowledgments}

We would like to acknowledge the KEKB accelerator group for excellent operation of the collider. I would also like to thank the conference organizers for their efforts. 

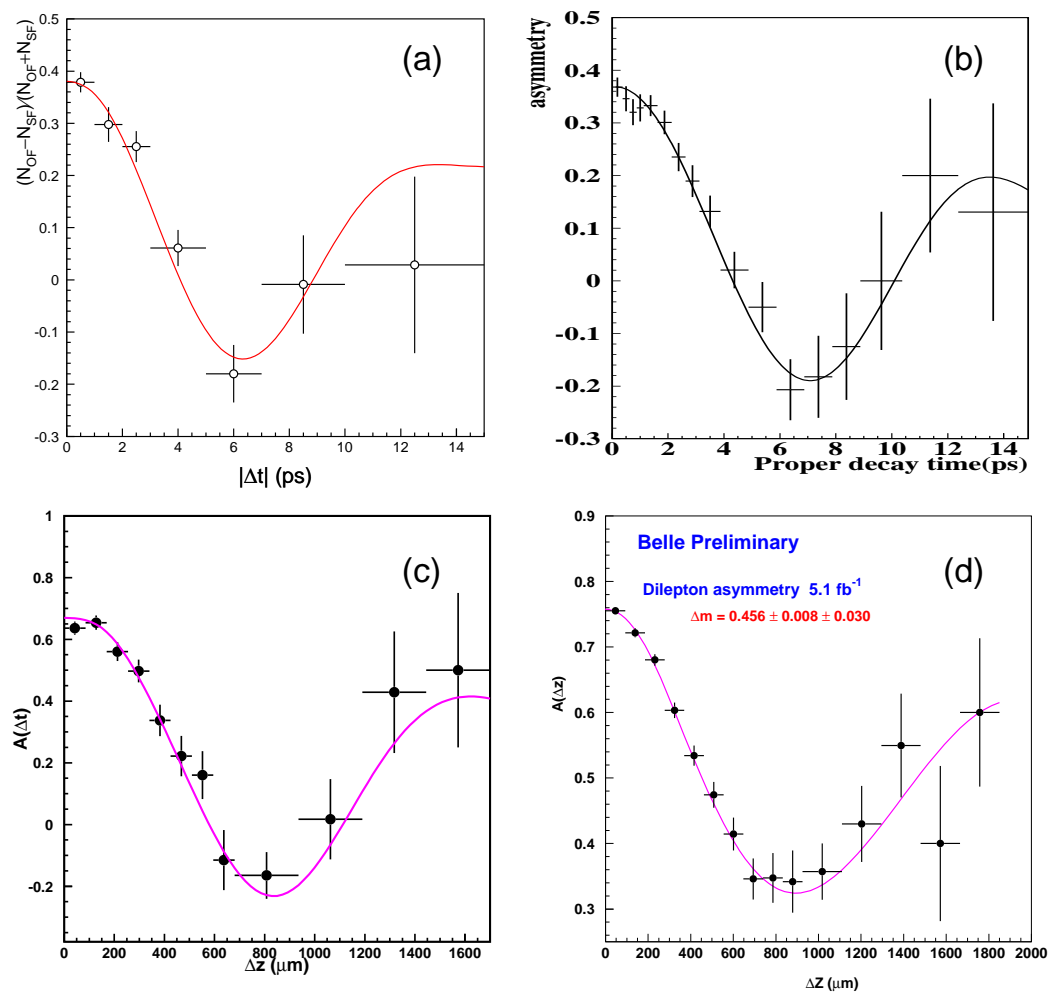

Figure 4: Asymmetry as a function of the proper decay time difference for (a) hadronic modes, (b) semi-leptonic mode, (c) partially reconstructed $B^{0} \rightarrow$ $D^{*} \pi$, and (d) dileptons. Fit curves are also shown. 


\section{References}

[1] M. Kobayashi and T. Maskawa, Prog. Theor. Phys. 49 (1973) 652.

[2] Belle Collaboration, K. Abe et al., Phys. Rev. Lett. 87 (2001) 091802.

[3] KEKB B Factory Design Report, KEK Report No. 95-1 (1995) (unpublished).

[4] Belle Collaboration, K. Abe et al., The Belle Detector, KEK Report 20004, to appear in Nucl. Instrum. Methods.

[5] H. Quinn and A.I. Sanda, Eur. Phys. Jour. C15, 626 (2000).

[6] Throughout this article, the inclusion of the charge conjugate mode is implied.

[7] Belle Collaboration, K. Abe et al., BELLE-CONF-0105, contributed to LP01, Rome, July 2001.

[8] G. C. Fox and S. Wolfram, Phys. Rev. Lett. 41, 1581 (1978).

[9] Belle Collaboration, K. Abe et al., hep-ex/0202009 (submitted to Phys. Rev. Lett.). The results are revised.

[10] Belle Collaboration, K. Abe et al., Phys. Rev. Lett. 86 (2001) 3228.

[11] D.E. Groom et al. (Particle Data Group), Eur. Phys. J. C15, 1 (2000).

[12] LEP B Lifetimes Working Group, see http://www.cern.ch/claires/lepblife.htm.

[13] LEP B Oscillations Working Group, see http://www.cern.ch/LEPBOSC/. 